\title{
THE BEST OBSERVABLES FROM THE POINT OF VIEW OF A MODEL MAKER
}

\author{
Best Observables \\ Francesca Matteucci (francesc@sissa.it) \\ Dipartimento di Astronomia \\ Universita' di Trieste \\ Via G. B. Tiepolo 11, 34100 Trieste, Italy
}

\begin{abstract}
We select and discuss the best observables to be used to constrain models of galactic chemical evolution. To this purpose, we discuss the observables in our Galaxy, which is the best studied system, as well as in external galaxies.
\end{abstract}

Keywords: Evolution of galaxies, Abundances, Star formation histories

\section{Introduction}

The "best observables" from the point of view of a chemical evolution model maker are those which allow us to significatively constrain the model parameters. In other words, the best observables can be reproduced only by a specific combination of the model parameters. In order to analyze these best observables we should describe first the main parameters entering chemical evolution models:

- the birthrate function, namely the star formation rate (SFR) and the initial mass function (IMF),

- the nucleosynthesis and stellar evolution, namely the yields,

- the gas flows (infall and outflow),

- the feed-back between supernovae (SNe) and the interstellar medium (ISM).

The still embryonic nature of our knowledge of these quantities forces us to describe them by means of some free parameters and to make some simplifying assumptions.

The free parameters used in models of galactic chemical evolution are:

- the expression for the SFR (the exponent of the gas density and the efficiency of star formation),

- the expression for the infall or outflow rates, 
- the amount of energy injected from SNe into the ISM which effectively heats the gas.

The stellar nucleosynthesis and the IMF, although still uncertain, can be taken by independent sources. Therefore they cannot be considered as completely free parameters. As a "golden rule" we suggest that, in general, a good model of galactic chemical evolution should reproduce a basic set of observables and the number of free parameters should be less than the number of observables that they can fit.

The main assumptions made in models of galactic chemical evolution are:

- the instantaneous recycling approximation (I.R.A.) (i.e. ignoring the stellar lifetimes),

- the homogeneous and instantaneous mixing approximation.

The I.R.A. is a poor approximation if one wants to follow the evolution of the abundances of chemical elements produced on long timescales such as $\mathrm{Fe}$ and $\mathrm{N}$ or if one wants to model the evolution of a region where the gas fraction is small $(<0.1)$. Therefore, most of chemical evolution models nowadays relax the I.R.A. whereas, on the other hand, the other assumption is still widely used, and is a good approximation if relatively small regions are considered.

In our discussion we will focuse on the observables in our Galaxy, which is the best studied system. Some of these observables turn out to be better than others in the sense that they allow us to really constrain the models, since they can be reproduced only by a specific combination of the various parameters. In order to identify the best observables from the point of view of a model maker in the next section we will describe in more detail the main observational constraints and their relation to the mechanisms of formation and evolution of the Galaxy.

For external galaxies the number of observables is smaller: we observe chemical abundances and gas distribution also in external spirals, whereas in ellipticals we can only measure integrated properties such as colours and spectra. We will try to envisage here which are the best observables also for these galaxies and what we expect to measure in the future.

\section{The observables in the Milky Way}

Let us take our Galaxy as an example. The Galaxy is a spiral system where four main stellar components can be envisaged: the stellar halo, 
the thick disk, the thin disk and the bulge. The stars in each of these components have different chemical and kinematical characteristics; in particular, the stars in the halo are the most metal poor $(<[\mathrm{Fe} / \mathrm{H}]>\sim$ -1.5 dex), they travel on elongated orbits and therefore tend to possess high radial velocities. Their scale height above the Galactic plane is of the order of $3 \mathrm{kpc}$. The stars in the thin disk are rotating in circular orbits around the Galactic center, have a scale height above the plane of the order of $200 \mathrm{pc}$ and average metallicity of $\langle[\mathrm{Fe} / \mathrm{H}]\rangle=-0.2$ dex. The thin disk contains also gas and dust and the gas is either neutral hydrogen $(\mathrm{HI})$ or molecular hydrogen $\left(H_{2}\right)$. The thick disk stars have characteristics intermediate between the halo and the thin disk stars, both chemically and kinematically. The average metallicity of the thick disk stars is $\langle[\mathrm{Fe} / \mathrm{H}]\rangle=-0.6$ dex and their scale height is around 920 pc. The bulge stars have metallicities in the range $-1.5 \leq[\mathrm{Fe} / \mathrm{H}] \leq$ +1.0 dex and kinematics more like that of the halo stellar population.

The basic observables for our Galaxy are:

- 1) the relative number of halo and disk stars in the solar neighbourhood (S.N.),

- 2) the metallicity distribution of stars in the halo, bulge and local disk,

- 3) the local present day mass function (PDMF),

- 4) the absolute solar abundances,

- 5) the age-metallicity relation(s),

- 6) the relative abundance ratios as functions of the relative metallicity (relative to the Sun),

- 7) the distribution of angular momentum per unit mass of the stars of the different Galactic components,

- 8) the present time infall rate, gas fraction and SN rates (Ia, Ib, II) in the S.N.,

- 9) the abundance gradients along the disk,

- 10) the distribution of gas (HI plus $H_{2}$ ) and SFR along the disk.

1) The ratio between the halo stars and the total number of stars in the S.N. is $\sim 0.03$ (Pagel and Patchett 1975). However, this number is probably underestimated and a more realistic value of this ratio is $\sim 0.1$ (Chiappini et al. 1997). Models which attempt to form the disk out of the gas shed by the halo tend to overestimate this ratio. 


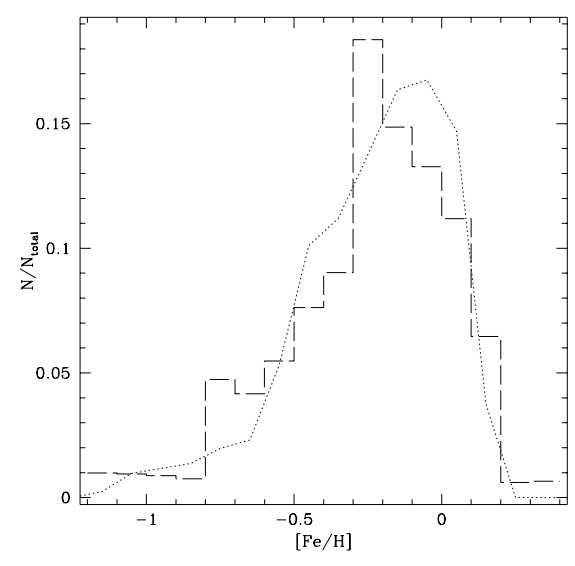

Figure 1. Observed and predicted G-dwarf metallicity distribution. The data are from Rocha-Pinto and Maciel (1996) whereas the model (continuous line) is from Chiappini e al. (1997).

Therefore, the indication is that the disk should have formed mainly out of extragalactic gas.

2) The $\mathrm{G}$ (or F)-dwarf metallicity distribution: there are less than $10 \%$ of stars with $[\mathrm{Fe} / \mathrm{H}]<-1.0$ dex and the distribution peaks at around -0.2 dex (see figure 1). This distribution is strictly related to the history of the star formation in the local disk, namely to the SFR and the IMF. The SFR, in turn, depends crucially on the mechanism of formation of the Galactic disk. A good fit of this distribution shows that the local disk formed by slow infall, in particular on a timescale of 6-8 Gyr (Chiappini et al. 1997; Boissier and Prantzos 1999). Slow infall is, in fact, the best solution to the G-dwarf problem (the fact that the Simple Model of chemical evolution predicts too many metal poor disk stars).

The metallicity distribution of halo stars is different from that of disk stars and indicates a formation for the halo faster than for the disk. Again, it is not possible to reproduce both distributions with a model implying that the disk forms out of the gas lost from the halo.

The metallicity distribution of stars in the Bulge shows a shape more similar to that of the halo stars but is skewed towards a much more metal rich domain (up to $[\mathrm{Fe} / \mathrm{H}] \sim+1.0 \mathrm{dex}$ ). This distribution (Mc William and Rich 1994) peaks at around $[\mathrm{Fe} / \mathrm{H}]=0.0$. Comparison with theoretical models shows that the Bulge formed faster than the disk and with a flatter IMF (Matteucci and Brocato, 1990; Matteucci et al. 1999).

3) The local PDMF is the distribution with mass of the local Main Sequence stars. It represents an important constraint, although very 


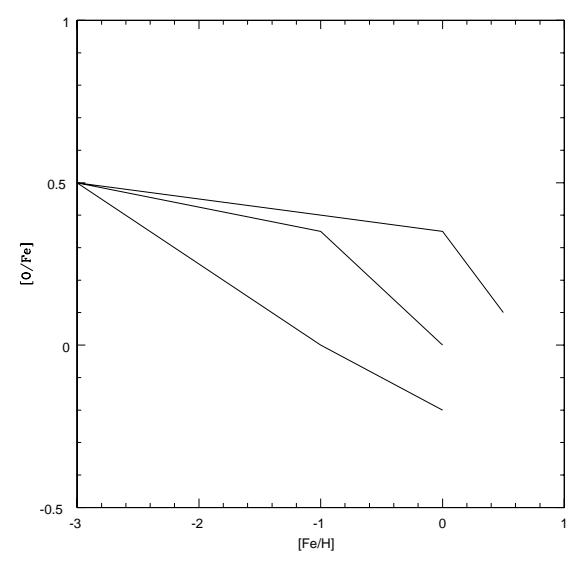

Figure 2. A sketch of the predicted behaviour of the $[\mathrm{O} / \mathrm{Fe}]$ ratio in the framework of the time-delay model for three different histories of star formation corresponding to: the upper curve with the longest plateau to the bulge, the median curve to the solar neighbourhood and the lower curve to magellanic irregular galaxies and/or the outermost regions of the Galactic disk.

few model makers take it into account, because it represents the convolution of the IMF and the SFR. A good model of chemical evolution should try to reproduce the PDMF since this guarantes that there is consistency between the IMF and the SFR. Most of the uncertainties in the PDMF reside in the low mass end, where data are lacking.

4) The solar and present time absolute abundances are known with good accuracy. However, the predicted abundances depend on all the model assumptions and therefore they can be reproduced by several combinations of model parameters. For this reason they do not represent an observable which can impose strong constraints on models.

5) The age-metallicity relation indicates that $[\mathrm{Fe} / \mathrm{H}]$ has continuosly increased with the galactic age, although the logarithmic nature of $[\mathrm{Fe} / \mathrm{H}]$ gives the impression of a flattening at late times. This observable contains many uncertainties due to the uncertain stellar ages (they can be wrong by a factor of two) and it is not a good constraint since it can be reproduced either by a closed-box model without I.R.A. or by an infall model with I.R.A.

In addition, a large spread in $[\mathrm{Fe} / \mathrm{H}]$ is observed at any fixed age and the nature of this spread is not yet clear. Therefore, we cannot learn much from the age-metallicity relation. Finally, one should rather speak of age-metallicity relations since it is very likely that different Galactic regions had different enrichment histories.

6) The abundance ratios versus metallicity ([el/Fe] vs $[\mathrm{Fe} / \mathrm{H}]$ ) are good observables. Abundance ratios depend only upon the stellar yields 


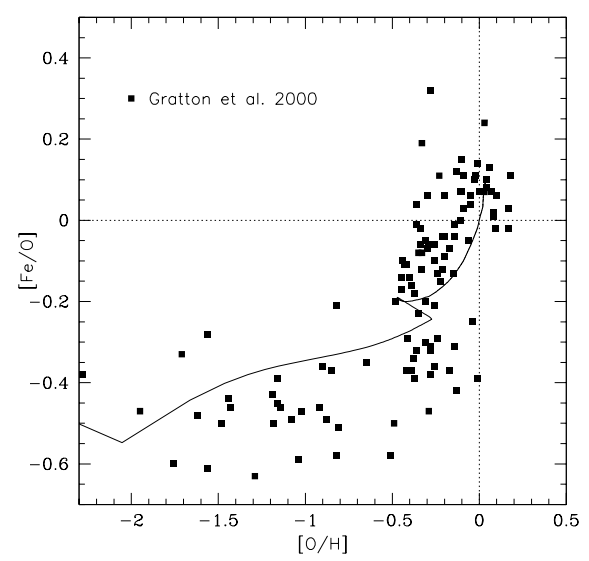

Figure 3. Observed and predicted $[\mathrm{Fe} / \mathrm{O}]$ versus $[\mathrm{O} / \mathrm{H}]$. The data are from Gratton et al. (2000) whereas the models are from Chiappini et al. (1997).

(IMF plus stellar nucleosynthesis) whereas the abundance ratios versus metallicity $([\mathrm{el} / \mathrm{H}]$ vs $[\mathrm{Fe} / \mathrm{H}])$ depend upon the yields and the star formation history (through $[\mathrm{Fe} / \mathrm{H}]$ ). Under the assumption of a constant IMF in space and time these relations can be interpreted in the framework of the time-delay between the enrichment due to SN II and SN Ia (time-delay model). A different star formation history, under this assumption, results in a different $[\mathrm{el} / \mathrm{Fe}]$ versus $[\mathrm{Fe} / \mathrm{H}]$ relation (see for example $[\mathrm{O} / \mathrm{Fe}]$ vs. $[\mathrm{Fe} / \mathrm{H}])$. As shown in figure 2, galaxies or Galactic regions with a slower evolutionary history show a change in the slope of the $[\mathrm{O} / \mathrm{Fe}]$ ratio occurring at smaller metallicities than in regions where the SFR has been quite fast (e.g. the Galactic bulge). Therefore, the fit of such diagrams gives us an indication about the star formation history of the Galaxy, besides the information on the nucleosynthesis and SN progenitors. An interesting aspect of these plots is that the $[\mathrm{Fe} / \mathrm{O}]$ vs. $[\mathrm{O} / \mathrm{H}]$ and $[\mathrm{Fe} / \mathrm{Mg}]$ vs. $[\mathrm{Mg} / \mathrm{H}]$ relations indicate that there has been a period between the formation of the halo and the disk when the star formation must have stopped. This effect is visible from the steep rise of the $[\mathrm{Fe} / \mathrm{O}]$ at $[\mathrm{O} / \mathrm{H}] \sim-0.2 \mathrm{dex}$, indicating that for a certain period (models indicate this period as no longer than 1 Gyr, Gratton et al. 2000) the Fe abundance was increasing whereas the O one was constant and this can be explained by a halt in the SFR. The same effect is present in the relation $[\mathrm{Fe} / \mathrm{Mg}]$ vs. $[\mathrm{Mg} / \mathrm{H}]$ (see Fuhrmann 1999)

Another interesting finding is by Nissen and Schuster (1997) who discovered some halo stars, which are likely to have formed in the outer halo, with metallicities overlapping those of disk stars $(-1.3 \leq[\mathrm{Fe} / \mathrm{H}] \leq$ 
$-0.5)$ but with $[\alpha / \mathrm{Fe}]$ ratios lower than the corresponding ratios in disk stars of the same metallicity. As a consequence of this, the transition between halo and disk was probably not a smooth one, as originally suggested by Eggen et al. (1962). A possible interpretation for these halo stars can be that the halo formed inside-out, namely that the external halo was formed by slow infall. An alternative explanation can be that these halo stars have been accreted from dwarf satellites of the Galaxy, where the star formation proceeded in bursts followed by quiescent periods during which only iron was produced.

7) The distribution of the angular momentum per unit mass as a function of the angular momentum (Wyse and Gilmore, 1992) shows that the halo and the bulge stars have a very similar distribution and the same holds for the stars in the thick and thin disk but the distribution of the halo-bulge is different from that of the thick-thin disk, clearly indicating that the bulk of the disk cannot have formed out of gas lost by the halo. As a consequence of this, we conclude that it is likely that most of the gas which formed the disk came from outside.

8) The present time infall rate, gas fraction and SN rates are good constraints only if coupled with all the others. In fact, theoretical models can always be adjusted to reproduce these absolute quantities by varying the various parameters, as in the case of the absolute abundances. However, the ratio between the SN rates is a good observable since it depends only on the supernova progenitors and the IMF.

9) Strong constraints on the mechanism of formation of the Galactic disk are imposed by the abundance gradients. The gradients show that the abundances of heavy elements tend to decrease with the galactocentric distance. Abundance gradients are strongly related to the mechanism of formation of the disk, in particular on the infall law and the star formation rate. It can be shown that the first condition required to fit the observed gradients (measured from HII regions, planetary nebulae, B stars) is to assume an "inside-out" formation for the Galactic disk, in the sense that the inner disk must have formed faster than the outer disk. The second condition is that the SFR should be strongly declining with the increasing galactocentric distance. Both these assumptions are necessary. Radial flows may enhance the predicted gradients but only under specific conditions. A particularly useful abundance gradient to measure is the $\mathrm{D}$ gradient, which is expected to be positive. This element, in fact, is only destroyed in stars (astration), so it is a good indicator about the star formation history along the disk. Gradients of abundance ratios such as $[\mathrm{O} / \mathrm{Fe}]$ and $[\mathrm{N} / \mathrm{O}]$ are important since they give us an idea about the timescales of disk formation at different galactocentric distances. A negative $[\mathrm{O} / \mathrm{Fe}]$ gradient, for example, would mean that the outer regions of the disk formed more slowly than the 
inner one thus allowing more pollution from type Ia SNe for the same $[\mathrm{Fe} / \mathrm{H}]$. On the other hand, a positive $[\mathrm{O} / \mathrm{Fe}]$ gradient would mean the contrary.

10) The conclusions above are valid also for reproducing the SFR and the gas distributions along the disk. These distributions cannot be reproduced unless a strongly varying SFR is assumed. This strong variation is achieved either by adding a dependence of the SFR on the total surface mass density or a dependence on $R^{-1}$ ( $R$ is the galactocentric distance (see Prantzos and Boissier, 1999). The dependence on the surface gas density which best fits the data is $k \sim 1.5$, in very good agreement with the observational estimate by Kennicutt (1998). The gas distribution along the disk shows a maximum at $\sim 4 \mathrm{kpc}$ followed by a rapid drop for smaller galactocentric distances, which can be better explained by including some dynamical effects such as the presence of a central bar (see Portinari and Chiosi 2000). Observationally, the SFR along the Galactic disk is a quite uncertain quantity derived from the distributions of pulsars, SN remnants, Lyman continuum photons and molecular clouds under the assumption of an IMF. Therefore, it is better to plot the ratio $S F R(R) / S F R\left(R_{\odot}\right)$ in order to avoid the uncertainty related with the choice of the IMF. The gas distribution along the disk is determined from the $\mathrm{HI}$ and $H_{2}$ gas. The distribution of this latter is quite uncertain since is derived by assuming a conversion factor (usually constant along the disk) between the amount of $\mathrm{CO}$ and the amount of $\mathrm{H}_{2}$.

\section{The observables in spiral galaxies}

In external spirals the most obvious observables are represented by the abundance gradients and the gas and SFR distributions, when available. Abundance gradients and gas distributions in external spirals are similar to those in the Milky Way (Henry and Worthey, 1999). This fact may indicate that galactic disks have a common origin (i.e. an inside-out formation) and that we can perhaps just describe the evolution of disks by using suitable scaling laws based on differences on the total and gas mass. Other observables in external spirals and in the Galaxy are the colour gradients along the disk. Prantzos and Boissier (2000) have shown that the assumption of an "inside-out" formation for disks can well reproduce the observed gradients including the absence of colour gradients at large galactocentric distances. In fact, this hypothesis implies different scale lengths for the distribution of stellar profiles. In particular, in the inside-out scenario the scale lenght in the $\mathrm{B}$ band is predicted to be $R_{B}=4 \mathrm{kpc}$ whereas that in the $\mathrm{K}$ band 
is $R_{K}=2.6 \mathrm{kpc}$, in good agreement with observations. This is due to the fact that in the inner regions of the disk one predicts considerably older stellar populations relative to the outer regions where there are mostly young stars. However, photometric models still contain many uncertainties mainly because of the existence of the age-metallicity degeneracy problem, consisting in the fact that age and metallicity act in the same way on integrated colors. Therefore, it is difficult to disentangle the two effects and integrated colors are not themselves good observables unless other constraints are considered at the same time.

\section{Observables in elliptical galaxies}

The observables which are relevant to study the chemical evolution of elliptical galaxies are represented by the metallicity indices, such as for example $\mathrm{Mg}_{2},\langle F e\rangle$ and $H_{\beta}$. These indices are measured from integrated spectra and depend on the metallicity and the age (again the age-metallicity degeneracy) of the stellar population which dominates in the visual light. The index $H_{\beta}$ perhaps is an exception since it depends mostly on the age and it can be used to break the degeneracy. The main problem with metallicity indicators is that they do not represent the real abundances and one needs to use a calibration to calculate such abundances. Elliptical galaxies show a mild increase of $M g_{2}$ with galactic mass (measured through the stellar velocity dispersion): this is known as "mass-metallicity" relation.

Data for cluster and field ellipticals have shown that the relation $<F e>$ vs. $M g_{2}$ is quite flat, indicating that the $[\mathrm{Mg} / \mathrm{Fe}]$ ratio should be an increasing function of the galactic luminosity. This finding is the contrary of what is expected from galactic models with SN-driven galactic winds, as originally proposed by Larson (1976). These models, in fact, predict exactly the opposite, due to the longer star formation period predicted for the more massive ellipticals relative to the less massive ones. An explanation for this behaviour can be that galactic winds are occurring earlier in massivegalaxies than in small galaxies and this can be achieved if the SFR increases with galactic mass. However, this trend of the SFR has to be calibrated in such a way not to destroy the mass-metallicity relation (see Matteucci 1994). The metallicity indices indicate the existence of abundance gradients also inside ellipticals but it is not yet clear if the gradient of $M g_{2}$ is flatter, steeper or the same as the gradient of $\langle F e\rangle$. This is an important point in order to understand the formation and evolution of these galaxies. Another strong constraint on the evolution and formation of ellipticals is provided by 
the abundances and abundance ratios measured in clusters of galaxies (see Matteucci 1996 for a review).

\section{Conclusions}

In this paper we have analyzed the best observables from the point of view of a model maker. Although we are not able to identify a unique model of galactic chemical evolution, we can envisage those observables which allow us to better constrain the models. We have discussed the available constraints for the Milky Way, external spirals and ellipticals and their interpretation by means of chemical evolution models. The comparison between models and observations suggests that the best observables are:

- i) The abundance ratios and the relation between abundance ratios and metallicity. They allow us to impose constraints on the stellar nucleosynthesis and on the star formation history, under the assumption of a constant IMF.

- ii) The distribution of dwarf stars as a function of metallicity. This is related to the star formation history and therefore to the evolution of the gas content. It would be auspicious to be able to observe such a distribution also in external galaxies, both spirals and ellipticals to gain insight onto their formation and evolution.

- iii) Kinematical and chemical studies of stars representing the halodisk transition provide a very important constraint in connection with the formation and evolution of the Galaxy. In particular, it is important to search for possible correlations between orbital parameters and abundance ratios.

- iv) The abundance gradients along galactic disks are sensitive to the star formation history and stellar nucleosynthesis. They can be used to infer the story of the formation of disks and gradients of abundance ratios between elements, formed on different timescales, can also give an idea about the timescales of disk formation at various galactocentric distances. Abundance gradients are now measured in external spirals and in elliptical galaxies (although in this case the metallicity is inferred through indices) and we hope that in the next years more and more data will be available. In particular, we would like to see detailed abundance measurements of elements such as $\alpha$-elements $(\mathrm{O}, \mathrm{Mg}, \mathrm{Si}, \mathrm{Ca})$ and $\mathrm{Fe}$ which represent an important tool for understanding the 
mechanisms of galaxy formation and evolution in external galaxies, since they can be used as cosmic clocks and are related to the star formation history. As a consequence, abundances and abundance ratios can also be used to infer the nature of high redshift galaxies.

\section{Acknowledgements}

I would like to thanks Cristina Chiappini and Donatella Romano for their contribution to the work describe here. This work has been partially supported by the Italian MURST, through COFIN98 at Padova.

\section{References}

Boissier, S., Prantzos, N. (1999) MNRAS 307, 857

Chiappini, C., Matteucci, F., Gratton, R. (1997) Astrophys. J. 477, 765

Eggen, Lynden-Bell, Sandage (1962) Astrophys. J. 136 , 748

Fuhrmann, K. (1999) Astrophys. Space Sci. 265 , 265

Gratton, R.G., Carretta, E., Matteucci, F., Sneden, C. (2000) Astron. Astrophys. 358, 671

Kennicutt, R.C. (1989) Astrophys. J. 498, 541

Henry, R.B.C., Worthey, G. (1999) PASP 111, 919

Larson, R.B. (1976) MNRAS 176, 31

Matteucci, F. (1991) in SN1987A and Other Supernovae, ed. by I.J. Danziger and K. Kjär, E.S.O. Publ. p.703

Matteucci, F. (1994) Astron. Astrophys. 288, 57

Matteucci, F. (1996) Fundametals of Cosmic Phys. Vol. 17, p.283

Matteucci, F., Brocato, E. (1990) Astrophys. J. 365, 539

Matteucci,F., Romano, D., Molaro, P. (1999) Astron. Astrophys. 352, 117

McWilliam, A., Rich, R.M. (1994) Astrophys.J. Suppl. 91, 749

Nissen, P.E., Schuster, W.J. (1997) Astron. Astrophys. 326, 751

Pagel, B.E.J., Patchett, B.C. (1975) MNRAS 172, 13

Portinari, L., Chiosi, C. (2000) Astron. Astrophys. , 355, 929

Prantzos, N., Boissier, S. (2000) MNRAS 313, 338

Rocha-Pinto, H., Maciel, W.J. (1996) MNRAS 279, 447

Wyse, R.F.G., Gilmore, G. (1992) Astron. J. 104, 144 
matteucci.tex; 1/01/2019; 8:03; p.12 